Discrete Comput Geom 37:267-286 (2007)

DOI: $10.1007 / \mathrm{s} 00454-006-1283-6$

\title{
Harmonic Algebraic Curves and Noncrossing Partitions*
}

\author{
Jeremy L. Martin, ${ }^{1}$ David Savitt, ${ }^{2}$ and Ted Singer ${ }^{3}$ \\ ${ }^{1}$ Department of Mathematics, University of Kansas, \\ 405 Snow Hall, 1460 Jayhawk Blvd., Lawrence, KS 66045, USA \\ jmartin@math.ku.edu \\ ${ }^{2}$ Department of Mathematics, University of Arizona, \\ 617 N. Santa Rita Ave., Tucson, AZ 85721, USA \\ savitt@math.arizona.edu \\ ${ }^{3} 38$ Greenacres, Scarsdale, NY 10583, USA \\ ted.singer@gmail.com
}

\begin{abstract}
Motivated by Gauss's first proof of the Fundamental Theorem of Algebra, we study the topology of harmonic algebraic curves. By the maximum principle, a harmonic curve has no bounded components; its topology is determined by the combinatorial data of a noncrossing matching. Similarly, every complex polynomial gives rise to a related combinatorial object that we call a basketball, consisting of a pair of noncrossing matchings satisfying one additional constraint. We prove that every noncrossing matching arises from some harmonic curve, and deduce from this that every basketball arises from some polynomial.
\end{abstract}

\section{Introduction}

The first proof of the Fundamental Theorem of Algebra was published in 1746 by d'Alembert [2]. In an attempt to correct the lack of rigor in d'Alembert's approach, as well as in subsequent attempts, Gauss offered a new proof in 1799 in his doctoral thesis [5]. Gauss's argument, while characteristically elegant, was itself not entirely satisfactory. Both d'Alembert's and Gauss's proofs were subsequently made completely rigorous (for the whole story, see, e.g., pp. 195-200 of [13]).

Gauss approached the problem by examining the real algebraic curves

$$
R=\{z: \operatorname{Re} f(z)=0\}, \quad I=\{z: \operatorname{Im} f(z)=0\},
$$

\footnotetext{
* The first author was supported in part by an NSF Postdoctoral Fellowship.
} 


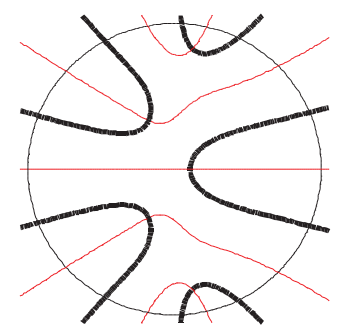

(a)

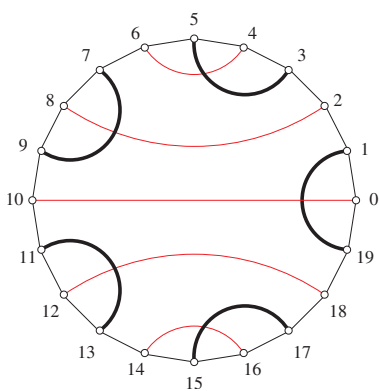

(b)

Fig. 1. (a) The curves $R$ (thick) and $I$ (thin) arising from $f(z)=z^{5}+6 z^{3}+3 z^{2}+5 z-2$, with a circle $S$ superimposed. (b) The associated combinatorial basketball.

in $\mathbb{R}^{2}$, where $f(z)$ is a complex polynomial of degree $n$. (See Fig. 1 for an example.) Note that the set of roots of $f$ is precisely $R \cap I$. For a suitably large disk $D$, each of the curves $R$ and $I$ meets the boundary circle $S=\partial D$ in exactly $2 n$ points, and it can easily be shown that the points of $R \cap S$ alternate with those of $I \cap S$ around $S$. Gauss argued that $R \cap D$ (resp. $I \cap D$ ) must consist of $n$ components and that each component of $R$ must cross some component of $I$ inside $D$; therefore, $\#(R \cap I) \geq n$.

We are interested in the topology of the curves $R$ and $I$, both singly and together. Much of the research on the topology of real plane algebraic curves (the topic of Hilbert's 16th problem) has focused on classifying the possible configurations of ovals (bounded connected components); see, e.g., [15]. However, the curves $R$ and $I$ are harmonic, and so the maximum principle [1, Theorem 21, p. 166] implies that they have no ovals at all. The topological information that can be extracted from the pair $R, I$ is of a different sort entirely.

Suppose that $R$ and $I$ are both nonsingular. (In particular, it is necessary that $f(z)$ have no repeated roots; as we will see, this condition is in a certain sense sufficient.) Labeling the $2 n$ points of $R \cap S$ cyclically and pairing off those points that lie in the same component of $R$ yields a noncrossing matching of order $n$. (Noncrossing partitions are an important subject in modern combinatorics; for an overview, see the excellent survey by Simion [10].) Repeating the construction for $I$ instead of $R$ yields a second noncrossing matching that is interlaced with the first. The matching obtained from $R$ must cross the matching obtained from $I$ exactly $n$ times, corresponding to the $n$ roots of the polynomial $f$. Two arbitrary interlaced noncrossing matchings of order $n$ must have at least $n$ crossings between them (Lemma 2.6), and may have more; if the number of such crossings is exactly $n$, we call this pair of noncrossing matchings a basketball of $\operatorname{order} n$. For example, Fig. 1(b) is the basketball

$$
\begin{gathered}
(\{\{0,10\},\{2,8\},\{4,6\},\{12,18\},\{14,16\}\}, \\
\{\{1,19\},\{3,5\},\{7,9\},\{11,13\},\{15,17\}\}) .
\end{gathered}
$$

It is natural to ask whether every basketball arises from a polynomial in this way. Our main result, the Inverse Basketball Theorem (Theorem 3.1), answers this question in the affirmative. The proof is constructive, and draws on elementary tools from combinatorics, 
topology, and complex analysis. The Inverse Basketball Theorem is very close in spirit to the Viro patchworking theorem [8], [14], and in fact can be viewed as a simplified version of Viro patchworking in the context of harmonic algebraic curves.

There is a natural bijection between basketballs of order $n$ and noncrossing partitions with $n$ blocks of size 4. It follows from a result of Edelman [4] that the basketballs are enumerated by the quasi-Catalan numbers $(1 /(3 n+1))\left(\begin{array}{c}4 n \\ n\end{array}\right)$. These numbers also count plane quaternary trees and dissections of a polygon into pentagons; there are natural bijections between basketballs and each of these combinatorial sets. The quasiCatalan numbers also enumerate certain plane trees (see [9]), although there is no obvious bijection involved.

A more subtle combinatorial-topological invariant of the polynomial $f$ arises from the curves

$$
C_{\theta}(f)=\left\{z: \operatorname{Im}\left(e^{-i \theta} f(z)\right)=0\right\},
$$

regarded as a family parameterized by $\theta \in \mathbb{R} / \pi \mathbb{Z}$. Note that $I=C_{0}(f)$ and $R=$ $C_{\pi / 2}(f)$. We will see that $C_{\theta}(f)$ is singular for only finitely many values of $\theta$. Therefore, we can study the family of noncrossing matchings, equipped with a cyclic order, obtained by letting $\theta$ vary; we call this family the necklace of matchings associated to $f$. Notice that the necklace depends only on the polynomial $f$ itself, and not on a choice of angle.

The paper is structured as follows. In Section 1 we give a modern exposition of Gauss's proof of the Fundamental Theorem of Algebra, and explain how Gauss's ideas may be used to associate basketballs with complex polynomials. Section 2 is devoted to the combinatorics of basketballs. Section 3 contains the proof of the Inverse Basketball Theorem. We conclude in Section 4 with some brief remarks on necklaces of matchings; there appears to be much more to say here from both the combinatorial and geometric points of view. Full-color figures may be found in the electronic version of this article at www.springerlink.com.

\section{Gauss's Proof of the Fundamental Theorem of Algebra}

We begin by describing Gauss's approach to the Fundamental Theorem of Algebra [5]; for the technical details, see [6]. Let $f(z)$ be a monic polynomial of degree $n$, and consider the two curves

$$
\begin{aligned}
R & =\{z: \operatorname{Re} f(z)=0\}, \\
I & =\{z: \operatorname{Im} f(z)=0\} .
\end{aligned}
$$

In polar coordinates $z=r e^{i \theta}$, the curves $R$ and $I$ are given by the equations

$$
\begin{aligned}
& \operatorname{Re} f(z)=r^{n} \cos n \theta+(\text { lower terms })=0, \\
& \operatorname{Im} f(z)=r^{n} \sin n \theta+(\text { lower terms })=0 .
\end{aligned}
$$

Let $S_{r}$ denote the circle $\{z:|z|=r\}$, and $D_{r}$ the disk $\{z:|z| \leq r\}$. By taking $r$ sufficiently large, we can ensure that

- $\operatorname{Re} f(z)$ has $2 n$ zeros on $S_{r}$, arbitrarily close (in angle) to the zeros of $\cos n \theta$; and

- $\operatorname{Im} f(z)$ has $2 n$ zeros on $S_{r}$, arbitrarily close to the zeros of $\sin n \theta$. 
That is, $R \cap S_{r}$ and $I \cap S_{r}$ each contain exactly $2 n$ points, and these $4 n$ points alternate around the circle. (See Fig. 1 for an example.) Suppose that $R$ and $I$ are both smooth curves. Then the intersection $R \cap D_{r}$ must contain $n$ disjoint $\operatorname{arcs} R_{1}, \ldots, R_{n}$, each of which joins two distinct points of $R \cap S_{r}$. Since these arcs do not cross, each arc $R_{i}$ must have an even number of points of $R \cap S_{r}$ on either side of it, by the Jordan Curve Theorem.

Likewise, $I \cap D_{r}$ contains $n$ disjoint arcs $I_{1}, \ldots, I_{n}$, each of which joins two distinct points of $I \cap S_{r}$. Since there is exactly one point of $I \cap S_{r}$ lying between each point of $R \cap S_{r}$, each arc $R_{i}$ must have an odd number of points of $I \cap S_{r}$ on either side of it. It follows that some arc $I_{j}$ joins a point on one side of $R_{i}$ with a point on the other, and therefore must intersect $R_{i}$. These $n$ intersection points, one for each $R_{i}$, are the roots of our polynomial $f$. (This step requires only the Intermediate Value Theorem, rather than the Jordan Curve Theorem, because Re $f(z)$ changes sign along the arc $I_{j}$.) This establishes:

Proposition 1.1. Let $f(z)$ be a monic polynomial such that the curves $R$ and $I$ are nonsingular. Then $f(z)$ has $n$ roots.

Gersten and Stallings [6] complete the proof of the Fundamental Theorem of Algebra by proving that if $f(z)$ has no roots, then $f(z)$ can be perturbed to obtain a new polynomial that still has no roots but for which $R$ and $I$ are nonsingular. We give a different approach.

Observe that the curve $I$ has $2 n$ asymptotes, with slopes at angles $k \pi / n$ for $k=$ $0, \ldots, 2 n-1$. Similarly, $R$ has asymptotes with slopes at angles $\left(k+\frac{1}{2}\right) \pi / n$. More generally, consider the family of real plane curves

$$
C_{\theta}(f)=\left\{z: \operatorname{Im}\left(e^{-i \theta} f(z)\right)=0\right\}
$$

parameterized by the circle $\mathbb{R} / \pi \mathbb{Z}$ (see Fig. 2). We often abuse notation by identifying $\theta \in \mathbb{R} / \pi \mathbb{Z}$ with its coset representative in the interval $[0, \pi)$. Note that $C_{\theta}(f)$ has $2 n$ asymptotes, with slopes at angles $(k \pi+\theta) / n$ for $k=0, \ldots, 2 n-1$. We regard $\arg (z)$ as an element in $\mathbb{R} / 2 \pi \mathbb{Z}$.

Lemma 1.2. The curve $C_{\theta}(f)$ is singular if and only if there exists $z \in C_{\theta}(f)$ such that $f^{\prime}(z)=0$.

Proof. If $f(z)=u(x, y)+i v(x, y)$ with $u, v$ real, then $C_{\theta}(f)$ is the curve defined by $v(x, y) \cos \theta-u(x, y) \sin \theta=0$. It follows that $\left(x_{0}, y_{0}\right) \in C_{\theta}(f)$ is a singular point if
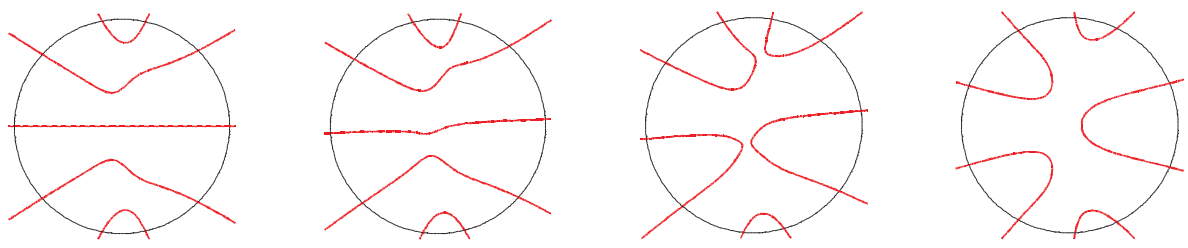

Fig. 2. The curves $C_{\theta}(f)$, where $f(z)$ is the quintic of Fig. 1 and $\theta=0, \pi / 12, \pi / 6, \pi / 2$, respectively. 
and only if $(\partial v / \partial x) \cos \theta-(\partial u / \partial x) \sin \theta$ and $(\partial v / \partial y) \cos \theta-(\partial u / \partial y) \sin \theta$ vanish at $\left(x_{0}, y_{0}\right)$. By the Cauchy-Riemann equations, it follows that the partial derivatives of $u$ and $v$ with respect to $x$ and $y$ all vanish at $\left(x_{0}, y_{0}\right)$, and therefore $z_{0}=x_{0}+i y_{0}$ is a zero of $f^{\prime}(z)$.

Corollary 1.3. If $f(z)$ has no repeated roots, then the curves $C_{\theta}(f)$ are singular for at most $n-1$ values of $\theta$. If $f(z)$ has a repeated root, then the curves $C_{\theta}(f)$ are all singular.

Proof. If $f^{\prime}(z)=0$ and $f(z) \neq 0$, then $z$ lies on $C_{\theta}(f)$ for exactly one value of $\theta \in \mathbb{R} / \pi \mathbb{Z}$, namely $\theta \equiv \arg (f(z))(\bmod \pi)$. On the other hand, if $f^{\prime}(z)=0$ and $f(z)=0$, then $z$ is a singular point on all $C_{\theta}(f)$.

We can now prove:

Theorem 1.4 (Fundamental Theorem of Algebra). Every polynomial $f(z)$ of degreen has exactly $n$ roots.

Proof. We may suppose that $f(z)$ is monic. If $f(z)$ has a repeated root, then it certainly has a root; by induction on the degree, $f(z)$ has exactly $n$ roots.

Assume, then, that $f(z)$ has no repeated roots. Choose any two different angles $\alpha, \beta \in \mathbb{R} / \pi \mathbb{Z}$ such that $C_{\alpha}(f)$ and $C_{\beta}(f)$ are nonsingular; this is possible by Corollary 1.3. Applying the argument which proved Proposition 1.1 to the curves $C_{\alpha}(f)$ and $C_{\beta}(f)$, the theorem follows.

The idea at the heart of Gauss's proof is that when $r$ is sufficiently large, the $2 n$ points of $C_{\alpha}(f) \cap S_{r}$ are paired via components of $C_{\alpha}(f)$. This associates to $f$ a purely combinatorial object: a matching, or partition into $n$ subsets of size 2 , of the set $C_{\alpha}(f) \cap S_{r}$. In a similar way, we can construct a matching on the set $C_{\beta}(f) \cap S_{r}$, and the existence of $n$ distinct roots follows from the combinatorial topology of these two matchings. In the remainder of this article, we study which (pairs of) such matchings can arise from polynomials. We begin by formalizing our study of the matchings which arise from the curves $C_{\theta}(f)$.

Suppose that $C_{\theta}(f)$ is nonsingular. By the maximum principle for harmonic functions, the curve $C_{\theta}(f)$ cannot have any bounded connected components. It follows that every connected component of $C_{\theta}(f)$ is diffeomorphic to $\mathbb{R}$. By Bézout's theorem, $C_{\theta}(f)$ cannot meet the quadratic curve $S_{r}$ in more than $2 n$ points. For $r$ sufficiently large, each connected component of $C_{\theta}(f)$ must meet $S_{r}$ in at least two points, so we deduce that $C_{\theta}(f)$ has at most $n$ connected components.

Recall, however, that the curve $C_{\theta}(f)$ has $2 n$ asymptotes, with slopes at angles $(k \pi+$ $\theta) / n$ for $k=0, \ldots, 2 n-1$. Each connected component approaches at most two of these asymptotes, and it follows that $C_{\theta}(f)$ has precisely $n$ connected components, each diffeomorphic to $\mathbb{R}$, and each approaching two of these asymptotes. Moreover, if $r$ is sufficiently large then each point of $C_{\theta}(f) \cap S_{r}$ lies on a different one of these asymptotes, 
so that which pairs of points $C_{\theta}(f) \cap S_{r}$ are joined by arcs in $C_{\theta}(f) \cap D_{r}$ is determined entirely by which pairs of asymptotes lie on the same connected component of $C_{\theta}(f)$.

If $C$ is any real plane curve, write $C(r)=C \cap D_{r}$. Suppose that $C(r)$ is nonsingular and has $m$ connected components, each with precisely two points lying on the circle $S_{r}$. Label these points counterclockwise from 0 to $2 m-1$, starting from the positive real axis. We make the following exception to the labeling rule: if $C=C_{0}(f)$ for some polynomial $f$ and $r$ is sufficiently large that $C(r)$ meets the component of $C$ asymptotic to the positive real axis, then we begin labeling from the point of $C(r)$ furthest along this asymptote.

Then $C(r)$ induces a matching $M(C, r)$ on the set $\{0, \ldots, 2 m-1\}$, where $a, b$ are matched if and only if the points labeled $a$ and $b$ lie on the same connected component of $C(r)$. If $C=C_{\theta}(f)$ is nonsingular and $r$ is sufficiently large, then we have observed that the matching $M\left(C_{\theta}(f), r\right)$ on $\{0, \ldots, 2 n-1\}$ does not depend on $r$; we denote this matching by $M(f, \theta)$. Our object is to study the matchings $M(f, \theta)$, as well as the pairs of matchings $(M(f, \alpha), M(f, \beta))$.

Remark 1.5. Recall that $f$ is assumed to be monic, so that if $C=C_{\theta}(f)$ and $\theta \in$ $[0, \pi)$, then for $r$ sufficiently large the point of $C \cap S_{r}$ which is labeled $k$ lies on the asymptote at angle $(k \pi+\theta) / n$.

Remark 1.6. If $C=C_{\theta}(f)$ is nonsingular, we say a few words for future reference about when $M(C, r)$ is defined, i.e., when each connected component of $C(r)$ has precisely two points lying on the circle $S_{r}$. Each connected component of $C$ is diffeomorphic to $\mathbb{R}$; since a connected component of $C(r)$ is a bounded connected subset of $C$, it is either a single point, or diffeomorphic to the interval $[0,1]$. Certainly each connected component which passes through the interior of the disk $D_{r}$ must intersect the boundary at least twice. It follows that $M(C, r)$ is well defined as long as $C$ has no point of tangency (either external or internal) to the circle $S_{r}$.

Remark 1.7. When $f(z)=z^{2}+b z+c$ is quadratic, the pair of matchings $(M(f, 0)$, $M(f, \pi / 2))$ is determined by the quadrant of the complex plane containing the discriminant $b^{2}-4 c$. An analogous description must exist for polynomials of higher degree. Indeed, regarding a monic polynomial of degree $n$ as a vector in $\mathbb{R}^{2 n}$ by taking the real and imaginary parts of its coefficients, the subset of $\mathbb{R}^{2 n}$ for which at least one of the curves $R$ and $I$ is singular is an algebraic set. Its complement, the set of polynomials for which $M(f, 0)$ and $M(f, \pi / 2)$ are both defined, is therefore a real semialgebraic set. However, every connected component of a real semialgebraic set is again semialgebraic [3, Theorem 2.4.5]; since the set of polynomials which yield a particular pair of matchings is a union of such connected components, that set is itself semialgebraic.

\section{Combinatorics of Basketballs}

Throughout, if $a \leq b$ are integers, we write $[a, b]$ for the set $\{a, a+1, \ldots, b-1, b\}$. (It should be clear from context whether this notation refers to an interval in $\mathbb{Z}$ or in $\mathbb{R}$.) 


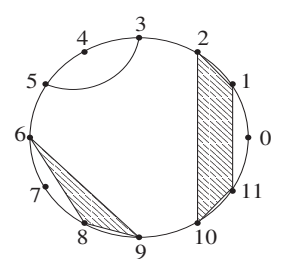

(a)

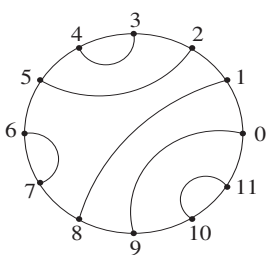

(b)

Fig. 3. (a) The noncrossing partition whose nonsingleton blocks are $\{1,2,10,11\},\{3,5\}$, and $\{6,8,9\}$. (b) A noncrossing matching.

Definition 2.1. Let $a \leq b$ be integers. A partition of $[a, b]$ is a collection of pairwise disjoint sets (called blocks) whose union is $[a, b]$. The number of blocks is the order of the partition. Two blocks are said to cross if there are integers $i<j<k<\ell$ such that $i, k$ belong to one block and $j, \ell$ belong to the other block. If no two blocks cross, then the partition is said to be noncrossing. A matching is a partition in which every block has cardinality 2 .

A noncrossing partition can be represented by placing the numbers $a, a+1, \ldots, b$ around a circle and connecting numbers in the same block, as in Fig. 3.

Example 2.2. If the curve $C_{\theta}(f)$ is nonsingular, then the matching $M(f, \theta)$ is noncrossing by the Jordan Curve Theorem.

The theory of noncrossing partitions comprises a substantial chapter in modern combinatorics; see [10] for a comprehensive survey. In the present study, we are concerned most with noncrossing matchings on the sets

$$
\mathbf{E}_{n}=\{0,2,4, \ldots, 4 n-2\} \quad \text { and } \quad \mathbf{O}_{n}=\{1,3, \ldots, 4 n-1\},
$$

where $n$ is a positive integer.

It is well known (and elementary to verify) that the number of matchings on $\mathbf{E}_{n}$ (resp. $\left.\mathbf{O}_{n}\right)$ is $(2 n-1)(2 n-3) \cdots(3)(1)=(2 n) ! / 2^{n} n$ !, and that the number of noncrossing matchings is the Catalan number $(1 /(n+1))\left(\begin{array}{c}2 n \\ n\end{array}\right)$.

Lemma 2.3. Let $M$ be a matching on [0,2n-1]. If $M$ is noncrossing, then exactly one member of each pair $\{i, j\}$ is even.

Proof. Suppose that $i<j$ and $i \equiv j \quad(\bmod 2)$. Then the sets

$$
X=[i+1, j-1], \quad Y=[0, i-1] \cup[j+1,2 n-1]
$$

both have odd cardinality, so some $x \in X$ is paired with some $y \in Y$. Then $\{x, y\}$ crosses $\{i, j\}$, contradicting the condition that $M$ is noncrossing.

Equivalently, if $M$ is a noncrossing matching on $\mathbf{E}_{n}$ or on $\mathbf{O}_{n}$, then every pair in $M$ consists of two numbers that are noncongruent modulo 4. 
Definition 2.4. A bimatching of order $n$ is a pair $B=\left(B^{\mathrm{e}}, B^{\mathbf{0}}\right)$, where $B^{\mathrm{e}}$ is a matching on $\mathbf{E}_{n}$ and $B^{\mathbf{0}}$ is a matching on $\mathbf{O}_{n}$. A pair in $B^{\mathbf{e}}$ (resp. $B^{\mathbf{0}}$ ) is called an even (resp. odd) pair of $B$. Let $M^{\mathbf{e}}, M^{\mathbf{0}}$ denote the matchings induced on [0,2n-1] by $B^{\mathbf{e}}, B^{\mathbf{0}}$ via the maps $[0,2 n-1] \rightarrow \mathbf{E}_{n}, \mathbf{O}_{n}$ sending $i \mapsto 2 n, 2 n+1$ respectively. We say that $B$ is the bimatching corresponding to the ordered pair of matchings $\left(M^{\mathrm{e}}, M^{\mathbf{0}}\right)$, and vice versa.

Example 2.5. Choose $\alpha, \beta \in[0, \pi)$ such that $C_{\alpha}(f)$ and $C_{\beta}(f)$ are nonsingular, and suppose that $\alpha<\beta$. Then we obtain a bimatching $B(f, \alpha, \beta)$ on $[0,4 n-1]$ corresponding to the pair of matchings $(M(f, \alpha), M(f, \beta))$,

This bimatching can be obtained geometrically as follows. For $r$ sufficiently large, label the points of $\left(C_{\alpha}(f) \cup C_{\beta}(f)\right) \cap S_{r}$ counterclockwise from 0 to $4 n-1$, beginning with the point of $C_{\alpha}(f)$ lying on the asymptote at angle $\alpha / n$. Again for $r$ sufficiently large, each point of $C_{\alpha}(f) \cap S_{r}$ will be labeled with an element of $\mathbf{E}_{n}$, and each point of $C_{\beta}(f) \cap S_{r}$ will be labeled with an element of $\mathbf{O}_{n}$. The bimatching is then induced by the $\operatorname{arcs}$ of $C_{\alpha}(f) \cap D_{r}$ and $C_{\beta}(f) \cap D_{r}$, respectively. See, e.g., Fig. 1.

Lemma 2.6. Let $B=\left(B^{\mathrm{e}}, B^{\mathbf{0}}\right)$ be a bimatching of order $n$. Suppose that both $B^{\mathrm{e}}$ and $B^{\mathbf{0}}$ are noncrossing. Then each pair of $B^{\mathbf{e}}\left(\right.$ resp. $\left.B^{\mathbf{0}}\right)$ crosses an odd number of pairs of $B^{\mathbf{0}}\left(\right.$ resp. $\left.B^{\mathbf{e}}\right)$.

Proof. Let $i, j$ be an odd pair of $B$ with $i<j$. Let

$$
X=\{i+1, i+3, \ldots, j-1\}, \quad Y=\{0,2, \ldots, i-1, j+1, \ldots, 4 n-2\} .
$$

Then a pair $k, \ell \in B^{\mathrm{e}}$ crosses $i, j$ if and only if exactly one of $k, \ell$ belongs to $X$. By Lemma 2.3, we have $i \not \equiv j \quad(\bmod 4)$, which implies that $\# X$ and $\# Y$ are both odd. Therefore there are an odd number of such pairs $k, \ell$. By the same argument, each pair in $B^{\mathbf{e}}$ crosses an odd number of pairs in $B^{\mathbf{0}}$.

In particular, each pair of $B$ crosses at least one pair of the opposite parity. We are interested primarily in the case when no extra crossings occur.

Definition 2.7. A basketball of order $n$ (or simply an $n$-basketball) is a bimatching $B=\left(B^{\mathbf{e}}, B^{\mathbf{0}}\right)$ of order $n$ in which the matchings $B^{\mathbf{e}}, B^{\mathbf{0}}$ are both noncrossing, and each pair $e \in B^{\mathrm{e}}$ crosses exactly one pair $o \in B^{\mathbf{0}}$. The pair of pairs $e, o$ is called a quartet. The set of all $n$-basketballs is denoted $\mathcal{B}_{n}$. (See Fig. 4.)

The bimatching $B(f, \alpha, \beta)$ of Example 2.5 is a basketball of order $n$. Indeed, every crossing between an even pair (i.e., an arc of $C_{\alpha}(f)$ ) and an odd pair (i.e., an arc of $\left.C_{\beta}(f)\right)$ corresponds to a root of $f$, and there are exactly $n$ roots.

Theorem 2.8. The number of basketballs of order $n$ is $(1 /(3 n+1))\left(\begin{array}{c}4 n \\ n\end{array}\right)$.

Proof. By a special case of Lemma 4.1 of [4], the given quantity enumerates the set $\mathcal{Q}_{n}$ of noncrossing partitions of [0,4n-1] into $n$ blocks of size 4 . We will exhibit a bijection $\varphi: \mathcal{B}_{n} \rightarrow \mathcal{Q}_{n}$. 


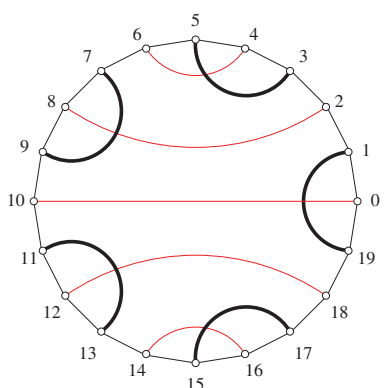

(a)

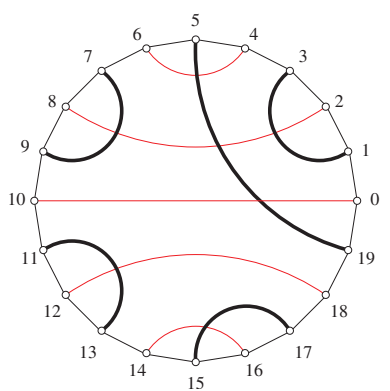

(b)

Fig. 4. (a) A basketball of order 5. (b) A bimatching that is not a basketball (the pair $\{5,19\}$ crosses three even pairs).

Let $B \in \mathcal{B}_{n}$. Define a partition $\varphi(B)$ of $[0,4 n-1]$ into $n$ blocks of size 4 by replacing each quartet $\{i, j\},\{k, \ell\}$ with the block $\{i, j, k, \ell\}$. It is elementary to deduce from the definition of a basketball that $\varphi(B)$ is noncrossing, hence belongs to $\mathcal{Q}_{n}$. It is also evident that the function $\varphi$ is injective.

Now let $Q \in \mathcal{Q}_{n}$ and let $K=\{a<b<c<d\}$ be a block of $Q$. Since $Q$ is noncrossing, each of the sets

$$
[a+1, b-1], \quad[b+1, c-1], \quad[c+1, d-1], \quad[d+1,4 n-1] \cup[0, a-1]
$$

must be a union of blocks, hence must have cardinality divisible by 4 . In particular, $a \equiv c \not \equiv b \equiv d \quad(\bmod 2)$. It follows that replacing $K$ with the two pairs $\{a, c\},\{b, d\}$, and doing the same for every other block of $Q$, yields an $n$-basketball $B$ such that $\varphi(B)=K$. Therefore $\varphi$ is surjective.

The numbers $(1 /(3 n+1))\left(\begin{array}{c}4 n \\ n\end{array}\right)$ form sequence A002293 in the On-Line Encyclopedia of Integer Sequences [11]. Other combinatorial objects enumerated in the same way include

- quaternary trees with $n$ internal nodes;

- dissections of a $(3 n+2)$-gon into $n$ pentagons; and

- rooted plane maps (see [9]).

Definition 2.9. Let $B$ be a basketball. An ear of $B$ is a quartet $\{i, j\},\{k, \ell\}$ such that the numbers $i, j, k, \ell$ are consecutive (in some order) modulo $4 n$.

For instance, the basketball shown in Fig. 5 has two ears: $\{4,6\},\{5,7\}$ and $\{15,17\}$, $\{16,18\}$. The following proposition reformulates in the language of basketballs the fact that any tree with at least two vertices has at least two leaves.

Proposition 2.10. Every basketball $B$ of order $n \geq 2$ has at least two ears.

Proof. We proceed by induction on $n$. The base case $n=2$ is amenable to proof by inspection, as there are only four basketballs of order 2 . 


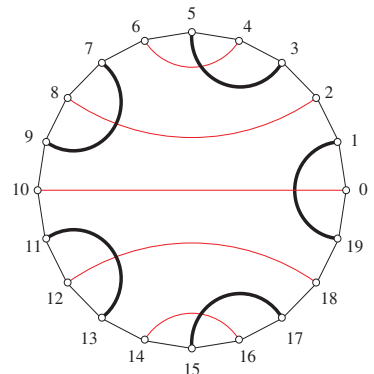

(a)

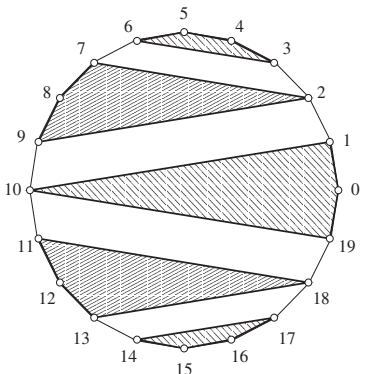

(b)

Fig. 5. The bijection $\varphi$ of Theorem 2.8, mapping (a) basketballs of order $n$ to (b) noncrossing matchings of $[0,4 n-1]$ with all blocks of size 4 . (Here $n=5$.)

Suppose now that $n>2$. Let $B$ be a basketball and let $T=\{i, j\},\{k, \ell\}$ be a quartet with $i<j<k<\ell$. If $T$ is an ear, then $B-T$ is a basketball on the ground set $[\ell+1,4 n-1] \cup[0, i-1]$. By induction, $B-T$ contains at least two ears $E, F$. Without loss of generality, we have $\ell+1 \notin E$. Then $E$ is an ear of $B$.

Now suppose that $T$ is not an ear. Then at least two of the four sets of (2.1) are nonempty. By a similar argument, each of those two sets contains an ear that is also an ear of $B$.

We point out several combinatorial problems related to bimatchings and basketballs.

(1) The set of $n$-basketballs is invariant under the operation of rotation: replacing each quartet $\{i, j\},\{k, \ell\}$ with $\{i-2, j-2\},\{k-2, \ell-2\}$, with all indices taken modulo $4 n$. Let $r(n)$ be the number of $n$-basketballs up to rotation, so that $(r(1), r(2), \ldots)=(1,2,6,22,103,614,3872, \ldots)$. These numbers occur as sequence A103941 in [11], enumerating unrooted loopless plane maps with $n$ edges [9, Theorem 4.4]. There does not seem to be an obvious relationship between plane maps and basketballs.

(2) More generally, the dihedral group $D_{4 n}$ (of order $8 n$ ) acts on the set of $n$ basketballs via its standard action on the plane. We refer to the nonsquare elements of the cyclic subgroup $C_{4 n}$ as half-rotations, since these transformations interchange $\mathbf{E}_{n}$ and $\mathbf{O}_{n}$. It would be of interest to count the number of orbits of $n$-basketballs under the action of $D_{4 n}$, and under the action of its various subgroups. In each case, by the Cauchy-Frobenius-Burnside Lemma of Polyá theory (see Section 7.24 of [12]) this problem reduces to counting the number of $n$-basketballs fixed by each element of $D_{4 n}$. Goldbach and Tijdeman [7] answer the analogous question for the actions of $C_{2 n}$ and $D_{2 n}$ on the set of noncrossing matchings.

(3) It would be interesting to enumerate the bimatchings of order $n$ by total number of crossings.

\section{The Inverse Basketball Theorem}

In light of Gauss's proof of the Fundamental Theorem of Algebra, it is natural to ask whether every basketball of order $n$ arises as $B(f, 0, \pi / 2)$ for some suitably chosen 
polynomial $f(z)$ of degree $n$. The main result of this section is that something more general is true.

Theorem 3.1. Fix any $\alpha, \beta \in[0, \pi)$ with $\alpha<\beta$. Then every basketball has the form $B(f, \alpha, \beta)$ for some monic polynomial $f$.

Before plunging into the details, we describe the argument informally. Proposition 2.10 suggests an inductive approach. Given an $n$-basketball $B$ for which we want to construct a realizing polynomial, we would like to remove an ear from $B$, inductively construct a realizing polynomial $f(z)$ for the resulting $(n-1)$-basketball $B^{\prime}$, and then insert the missing ear by replacing $f(z)$ with $f(z)(z-R)$ for some suitable $R$. If we choose $R$ to be much greater in absolute value than any of the roots of $f$, then the components of $B^{\prime}$ will be perturbed only slightly, and will retain their combinatorial structure near the origin, where $\arg (z-R) \approx \pi$ is close to $0 \quad(\bmod \pi)$. (No confusion should arise between the root $R$ and the curve $R=\{z: \operatorname{Re} f(z)=0\}$, which does not appear in this section.)

Example 3.2. Let $f(z)=z^{3}+i z^{2}+z-2$, a cubic whose associated basketball is shown in Fig. 6(a). Every root of $f(z)$ has complex magnitude $<2$. If we choose a new root that is much larger in magnitude, say $R=8+8 i$, then the basketball of $f(z)(z-R)$ is given combinatorially by "inserting an ear at $R$," as shown.

The following theorem explains how $M(f(z)(z-R), \theta)$ is obtained from $M(f, \theta)$, and is the crucial result from which Theorem 3.1 will follow. We first introduce some useful notation. If $M$ is a matching on [0,2n-1], define a matching $\widehat{M}$ on $[0,2 n+1]$ by

$$
\widehat{M}=\{\{0,2 n+1\}\} \cup\{\{i+1, j+1\}:\{i, j\} \in M\} .
$$

Also, for any positive real $R$, let $g_{R}(z)=(z-R) f(z)$.

Theorem 3.3. Let $f(z)$ be a monic polynomial, and let $\theta \in(0, \pi)$ such that $C_{\theta}(f)$ is nonsingular. Then, for $R$ sufficiently large, we have

$$
M\left(g_{R}, \theta\right)=\widehat{M(f, \theta)} .
$$

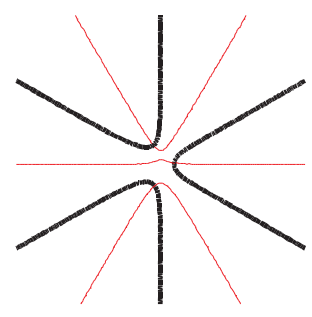

(a)

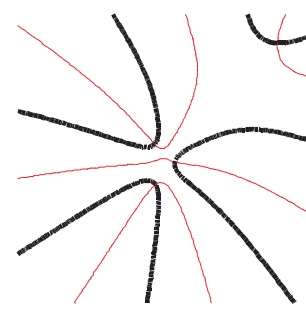

(b)

Fig. 6. (a) The basketball of $f(z)$, where $f(z)$ is the cubic of Example 3.2. (b) The basketball of $f(z)(z-R)$. 
Before we can prove Theorem 3.3, we must develop several subsidiary results. The first of these is the following, which is implicit in the statement of Theorem 3.3.

Proposition 3.4. Under the assumptions of Theorem 3.3, the curves $C_{\theta}\left(g_{R}\right)$ are nonsingular for $R$ sufficiently large.

To establish this fact, we first need the following technical result. The idea is that as $R \rightarrow \infty$, one root of $g_{R}^{\prime}(z)$ increases without bound, while the other $n-1$ roots approach the roots of $f^{\prime}(z)$.

Lemma 3.5. Let $\alpha_{1}, \ldots, \alpha_{n-1}$ denote the roots of $f^{\prime}(z)$. For $R \in \mathbb{R}$ sufficiently large, there is an ordering $\beta_{1}(R), \ldots, \beta_{n}(R)$ of the roots of $g_{R}^{\prime}(z)$ with the following properties: ${ }^{1}$

(1) if $i \leq n-1$, then $\beta_{i} \rightarrow \alpha_{i}$ as $R \rightarrow \infty$;

(2) $\left|\beta_{n}\right| \rightarrow \infty$ and $\arg \left(\beta_{n}\right) \rightarrow 0$ as $R \rightarrow \infty$;

(3) if $i \leq n-1$, then $\arg \left(g_{R}\left(\beta_{i}\right)\right) \rightarrow \arg \left(f\left(\alpha_{i}\right)\right)+\pi$ as $R \rightarrow \infty$;

(4) $\arg \left(g_{R}\left(\beta_{n}\right)\right) \rightarrow \pi$ as $R \rightarrow \infty$.

Proof. Note that $g_{R}^{\prime}(z)=f(z)+(z-R) f^{\prime}(z)$. Put $t=1 / R$, so that

$$
g_{R}^{\prime}(z) / R=(t z-1) f^{\prime}(z)+t f(z) .
$$

Note that $\lim _{t \rightarrow 0} g_{R}^{\prime}(z) / R=-f^{\prime}(z)$. The existence of an ordering of the roots with property (1) now follows from the argument principle [1, Theorem 18, p. 152]. Assertion (3) is evident: we have seen that $\beta_{i}(R)$ is bounded, and therefore that $\arg \left(\beta_{i}(R)-R\right) \rightarrow \pi$ as $R \rightarrow \infty$.

For properties (2) and (4), observe from the coefficients of $z^{n}$ and $z^{n-1}$ in $g_{R}^{\prime}$ that the sum of the roots of $g_{R}^{\prime}(z)$ is

$$
\frac{n}{n+1}\left(R-a_{n-1}\right)
$$

where $a_{n-1}$ is the coefficient of $z^{n-1}$ in $f(z)$. Since the sum $\beta_{1}+\cdots+\beta_{n-1}$ is bounded, so is

$$
\beta_{n}(R)-\frac{n}{n+1} R=-\left(\frac{n}{n+1} a_{n-1}+\beta_{1}+\cdots+\beta_{n-1}\right) .
$$

That is, $\beta_{n}(R)$ is within a bounded distance of $(n /(n+1)) R$, and so we have

$$
\arg \left(\beta_{n}\right) \rightarrow 0, \quad \arg \left(\beta_{n}-R\right) \rightarrow \pi, \quad \text { and } \quad\left|\beta_{n}\right| \rightarrow \infty,
$$

whence

$$
\arg \left(g_{R}\left(\beta_{n}\right)\right)=\arg \left(\left(\beta_{n}-R\right) f\left(\beta_{n}\right)\right) \rightarrow \pi
$$

Proof of Proposition 3.4. For $R$ sufficiently large, $g_{R}(z)$ has no repeated roots. By Lemma 3.5, we see that $\arg \left(g_{R}\left(\beta_{i}(R)\right)\right)$ becomes arbitrarily close to $\arg \left(f\left(\alpha_{i}\right)\right)+\pi$ for

\footnotetext{
${ }^{1}$ For simplicity of notation, we often abbreviate $\beta_{i}(R)$ by $\beta_{i}$.
} 
$i<n$, and to $\pi$ for $i=n$. Using the hypotheses that $C_{\theta}(f)$ is nonsingular and $\theta \neq 0$, it follows that $\arg \left(g_{R}\left(\beta_{i}(R)\right)\right)$ is not congruent to $\theta \quad(\bmod \pi)$ for any $i$, and so $C_{\theta}\left(g_{R}\right)$ is nonsingular for $R$ sufficiently large.

The next step in proving Theorem 3.3 is to show that for sufficiently large $R$, the topological behavior of the curve $C_{\theta}\left(g_{R}\right)$ inside a suitable disk $D_{r}$ is the same as that of the curve $C_{\theta}(f)$; that is, the passage from $f(z)$ to $g_{R}(z)$ preserves the combinatorial type of the matching $M(f, \theta)$.

Proposition 3.6. Let $f(z)$ be a monic polynomial of degree $n$, let $\theta \in(0, \pi)$ be such that $C_{\theta}(f)$ is nonsingular, and choose $r$ so that $C_{\theta}(f)$ is not tangent to the circle $S_{r}$. (Hence $M\left(C_{\theta}(f), r\right)$ is well defined by Remark 1.6.) Then for $R$ sufficiently large, we have

$$
M\left(C_{\theta}\left(g_{R}\right), r\right)=M\left(C_{\theta}(f), r\right) .
$$

Moreover, as $R \rightarrow \infty$, the point labeled $i$ on $C_{\theta}\left(g_{R}\right) \cap S_{r}$ approaches the point labeled $i$ on $C_{\theta}(f) \cap S_{r}$. In particular, if $r$ is sufficiently large, then for $R$ sufficiently large we have

$$
M\left(C_{\theta}\left(g_{R}\right), r\right)=M(f, \theta) .
$$

In order to prove Proposition 3.6, we will need the following (presumably standard) facts from metric topology.

Lemma 3.7. Let $(X, d)$ be a compact, locally connected metric space. For $x \in X$ and $\varepsilon>0$, write $B(x, \varepsilon)$ for the open ball of radius $\varepsilon$ centered at $x$. For any subset $K$ of $X$ and any $\varepsilon>0$, define

$$
N_{\varepsilon}(K)=\bigcup_{x \in K} B(x, \varepsilon) .
$$

For $\beta>0$ and $t \in(0, \beta)$, let $h_{t}: X \rightarrow \mathbb{R}$ be a family of continuous functions converging pointwise (hence uniformly) to some continuous $f: X \rightarrow \mathbb{R}$ as $t \rightarrow 0$. Define

$$
\begin{aligned}
Z & =\{x \in X: f(x)=0\}, \\
Y_{t} & =\left\{x \in X: h_{t}(x)=0\right\} .
\end{aligned}
$$

Then:

(1) For all $\varepsilon>0$, ift is sufficiently close to 0 then $Y_{t} \subset N_{\varepsilon}(Z)$.

(2) Suppose furthermore that $X \subset \mathbb{R}^{2}$, that $U \subset X$ is an open set on which the functions $f, h_{t}$ are harmonic, and that $f$ is nowhere locally identically zero on $U$. Then, for all $\varepsilon>0$, there exists $t_{0}=t_{0}(\varepsilon)>0$ such that $Z \cap U \subset N_{\varepsilon}\left(Y_{t} \cap U\right)$ for all $t<t_{0}$.

Proof. Suppose that (1) fails: that is, there exist $\varepsilon>0$ and sequences $t_{n} \rightarrow 0, y_{n} \in Y_{t_{n}}$ such that for all $n$ we have $B\left(y_{n}, \varepsilon\right) \cap Z=\emptyset$. Since $X$ is compact, the sequence $\left\{y_{n}\right\}$ has 
a limit point; therefore, replacing $y_{n}$ and $t_{n}$ by suitable subsequences, we may assume without loss of generality that the sequence $y_{n}$ converges to a point $y$.

Note that $y \notin Z$. Indeed, $B(y, \varepsilon / 2) \subset B\left(y_{n}, \varepsilon\right)$ for $n$ sufficiently large, so in fact $B(y, \varepsilon / 2) \cap Z=\emptyset$. Choose a connected neighborhood $U \subset B(y, \varepsilon / 2)$ of $y$; by the Intermediate Value Theorem, $f$ is either strictly positive or strictly negative on $U$. Since $f$ is continuous and $X$ is compact, we find a neighborhood $V \subset U$ of $y$ and a constant $\delta>0$ such that

$$
|f(x)|>\delta>0 \quad \text { for all } \quad x \in V .
$$

Now, choose $n$ sufficiently large so that $y_{n} \in V$ and (by uniform convergence) so that $\left|g_{t_{n}}(x)-f(x)\right|<\delta / 2$ for all $x \in X$. Then $f\left(y_{n}\right)>\delta$ by (3.1). On the other hand, $g_{t_{n}}\left(y_{n}\right)=0$, so $\left|f\left(y_{n}\right)\right|=\left|g_{t_{n}}\left(y_{n}\right)-f\left(y_{n}\right)\right|<\delta / 2$. This is a contradiction.

To prove (2), fix $\varepsilon>0$ and cover $Z \cap U$ with finitely many open balls $B_{1}, \ldots, B_{n}$ of radius $\varepsilon / 2$. (To do so, first cover $Z$ by finitely many such balls, and then discard the ones that do not meet $Z \cap U$.)

For each $i=1, \ldots, n$, the ball $B_{i}$ contains a point $z_{i}$ of $Z \cap U$. By the maximum principle [1, Theorem 21, p. 166] applied in a neighborhood of $z_{i}$ contained in $B_{i} \cap U$, we can find points $p_{i}, q_{i}$ in $B_{i} \cap U$ such that $f\left(p_{i}\right)<f\left(z_{i}\right)=0<f\left(q_{i}\right)$. Choose $\delta>0$ such that $\delta<\left|f\left(p_{i}\right)\right|,\left|f\left(q_{i}\right)\right|$ for all $i$.

By uniform convergence, we can choose $t_{0}=t_{0}(\varepsilon)>0$ so that

$$
\left|h_{t}(x)-f(x)\right|<\frac{\delta}{2} \quad \text { for all } \quad t \in\left(0, t_{0}\right), \quad x \in X .
$$

In particular,

$$
h_{t}\left(p_{i}\right)<-\frac{\delta}{2}<0<\frac{\delta}{2}<h_{t}\left(q_{i}\right)
$$

for all $i=1, \ldots, n$ and $t \in\left(0, t_{0}\right)$. Since the function $h_{t}$ is continuous, it has a root $w_{i} \in B_{i} \cap U$ for every $i$ (for example, along any path joining $p_{i}$ and $q_{i}$ ).

Now, for any $z \in Z \cap U$, we can find $i \in\{1, \ldots, n\}$ such that $z \in B_{i}$. Since also $w_{i} \in B_{i}$, we have $\left|z-w_{i}\right|<\varepsilon$ as desired.

Remark 3.8. Under the hypotheses of part (2) of Lemma 3.7, the conclusions of parts (1) and (2) together imply that the Hausdorff distance between $Z$ and $Y_{t}$, namely $\max \left(\inf \left(\varepsilon>0: Z \subset N_{\varepsilon}(Y)\right), \inf \left(\varepsilon>0: Y \subset N_{\varepsilon}(Z)\right)\right)$, tends to zero as $t \rightarrow 0$. Of course, part (2) of Lemma 3.7 holds for any family of functions $\left\{h_{t}\right\}, f$ that satisfy the maximum principle, even if they are not harmonic.

Proof of Proposition 3.6. By Lemma 3.5, the curves $C_{\theta}\left(g_{R}\right)$ are nonsingular for $R$ sufficiently large, say $R>1 / \beta$. Let $t=1 / R$, and write

$$
H_{t}(z)=g_{R}(z) / R=(t z-1) f(z) .
$$

(Note that $H_{t}(z)$ is well defined for $t=0$.) Then $\left\{C_{\theta}\left(H_{t}\right)\right\}_{t \in[0, \beta)}$ is a family of nonsingular real algebraic curves for $t \in[0, \beta)$, and $C_{\theta}\left(H_{t}\right)=C_{\theta}\left(g_{R}\right)$. Let $m$ be the number of connected components of $C_{\theta}(f)$. For $0 \leq j \leq 2 m-1$, let $P_{j}$ denote the point of $C_{\theta}(f)(r)$ that is labeled $j$. Let $h_{t}(x, y)$ be the real polynomial $\operatorname{Im}\left(e^{-i \theta} H_{t}(x+i y)\right)$. 
We will eventually apply Lemma 3.7 to the family $\left\{h_{t}\right\}$ on the domain $X=D_{r}$. Accordingly, we define

$$
\begin{aligned}
Y_{t} & =C_{\theta}\left(H_{t}\right) \cap D_{r}=C_{\theta}\left(g_{R}\right) \cap D_{r}, \\
Z & =C_{\theta}(f) \cap D_{r} .
\end{aligned}
$$

Claim. For $t$ sufficiently small, $Y_{t}$ has exactly $2 m$ points on the circle $S_{r}$.

To see this, consider the holomorphic function $\lambda_{t}(z)=h_{t}(r \cos z, r \sin z)$. The real zeros $(\bmod 2 \pi)$ of $\lambda_{t}(z)$ correspond exactly to the zeros of $h_{t}(x, y)$ on the circle $S_{r}$. For $\delta$ sufficiently small and $\alpha \in \mathbb{R}$ a nonroot of $\lambda_{0}$, the set

$$
T=\{z: \operatorname{Re}(z) \in[\alpha, \alpha+2 \pi], \operatorname{Im}(z) \in[-\delta, \delta]\}
$$

contains exactly one representative $(\bmod 2 \pi)$ of each of the $2 m$ real roots of $\lambda_{0}(z)$, and no other roots of $\lambda_{0}(z)$. Observe that the hypothesis that $C_{\theta}(f)$ is not tangent to the circle $S_{r}$ is precisely equivalent to the statement that the real zeros of $\lambda_{0}(z)$ are all simple zeros.

For $t$ sufficiently small, by the argument principle $\lambda_{t}(z)$ also has exactly $2 m$ roots in $T$. (Roots on the boundary of $T$ are ruled out by the uniform convergence of $\lambda_{t} \rightarrow \lambda_{0}$.) Hence $\lambda_{t}(z)$ has at most $2 m$ real roots $(\bmod 2 \pi)$. However, again by the argument principle, for any sufficiently small $\varepsilon>0$, if $t$ is sufficiently small then the disk of radius $\varepsilon$ around a real root of $\lambda_{0}(z)$ contains exactly one root of $\lambda_{t}(z)$. Since $\lambda_{t}(z)=0$ if and only if $\lambda_{t}(\bar{z})=0$, these roots of $\lambda_{t}(z)$ must be real. This establishes the claim; in fact, we have shown that as $R \rightarrow \infty$, the point $Q_{j}$ labeled $j$ on $Y_{t}$ approaches the point labeled $j$ on $Z$. (See Fig. 7.)

Let $C_{1}, \ldots, C_{m}$ denote the connected components of $C_{\theta}(f)$, and let $\Delta>0$ be smaller than the distance between any two of the components: that is, if $z_{j} \in C_{j}$ and $z_{k} \in C_{k}$ with $j \neq k$, then $\left|z_{j}-z_{k}\right|>\Delta$. Take $R$ sufficiently large, as above, so that $\left|P_{j}-Q_{j}\right|<\Delta / 2$ for all $j$.

Let $\varepsilon=\Delta / 3$, and further take $t=1 / R$ sufficiently small so as to satisfy both parts of Lemma 3.7. Note that the connected components of $N_{\varepsilon}(Z)$ are precisely $N_{\varepsilon}\left(C_{1}\right), \ldots$, $N_{\varepsilon}\left(C_{m}\right)$.

Let $U=\{z:|z|<r\}$, and fix points $u_{j} \in C_{j} \cap U$ for $j=1, \ldots, m$. By part (2) of Lemma 3.7, there exist points $v_{1}, \ldots, v_{m} \in Y_{t} \cap U$ such that $\left|u_{j}-v_{j}\right|<\varepsilon$. That is, $v_{j} \in N_{\varepsilon}\left(C_{j}\right)$. In particular, the $v_{j}$ are all distinct.

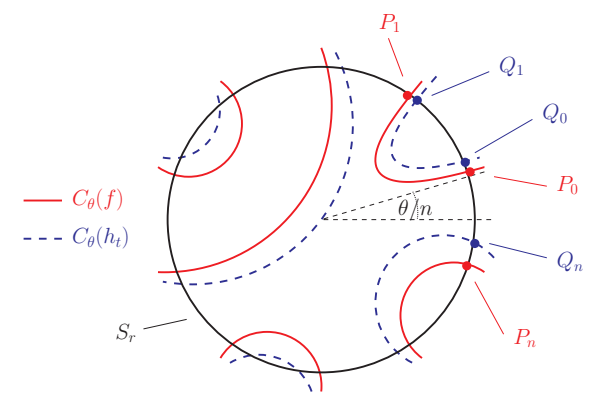

Fig. 7. How the curve $C_{\theta}\left(h_{t}\right)$ approaches the curve $C_{\theta}(f)$. 
Let $C_{j}^{\prime}$ be the connected component of $Y_{t}$ that contains $v_{j}$; then $C_{j}^{\prime} \subset N_{\varepsilon}(Z)$ by part (1) of Lemma 3.7. Since $C_{j}^{\prime}$ is connected, we have $C_{j}^{\prime} \subset N_{\varepsilon}\left(C_{j}\right)$. In particular, the $C_{j}^{\prime}$ are all different. Since $Y_{t}$ has exactly $2 m$ points on $|z|=r$, the $C_{j}^{\prime}$ exhaust all of the connected components of $Y_{t}$. Finally, since $\left|P_{i}-Q_{i}\right|<\Delta / 2$, it must be the case that $P_{i}$ lies on $C_{j}$ if and only if $Q_{i}$ lies on $C_{j}^{\prime}$. It follows that $M\left(C_{\theta}(f), r\right)=M\left(C_{\theta}\left(g_{R}\right), r\right)$.

We have now collected all the tools we need to prove Theorem 3.3, and thereby to characterize the combinatorial relationship between the matchings $M\left(g_{R}, \theta\right)$ and $M(f, \theta)$.

Proof of Theorem 3.3. For any $\delta>0$, we may choose $r$ sufficiently large that

- $M\left(C_{\theta}(f), r\right)$ is well defined and equal to $M(f, \theta)$,

- $\left|\arg \left(f(z) / z^{n}\right)\right|<\delta$ for $|z| \geq r$, and

- if $P_{0}, \ldots, P_{2 n-1}$ denote the points of $M\left(C_{\theta}(f), r\right)$ labeled $0, \ldots, 2 n-1$, respectively, then $\left|\arg \left(P_{k}\right)-(k \pi+\theta) / n\right|<\delta /(2(n+1))$ for all $k$.

By Proposition 3.6, we may then choose $R$ sufficiently large that

- $M\left(C_{\theta}\left(g_{R}\right), r\right)=M(f, \theta)$, and

- if $Q_{0}, \ldots, Q_{2 n-1}$ denote the points of $M\left(C_{\theta}\left(g_{R}\right), r\right)$ labeled $0, \ldots, 2 n-1$, respectively, then $\left|\arg \left(Q_{k}\right)-\arg \left(P_{k}\right)\right|<\delta /(2(n+1))$ for all $k$.

It follows from these properties that $\arg \left(g_{R}(z)\right)$ differs from $n \arg (z)+\arg (z-R)$ by at most $\delta$ if $|z| \geq r$ and $z \neq R$, and that $\left|\arg \left(Q_{k}\right)-(k \pi+\theta) / n\right|<\delta /(n+1)$.

Recall that $\theta$ is assumed to lie in the interval $(0, \pi)$. We define the interval $I_{k}(\delta)$ for $0 \leq k \leq 2 n$ as follows:

$$
I_{k}(\delta)= \begin{cases}\left(\frac{k \pi+\theta+\delta}{n+1}, \frac{k \pi+\theta-\delta}{n}\right) & \text { for } 0 \leq k \leq n-1, \\ \left(\frac{n \pi+\theta+\delta}{n+1}, \pi\right) & \text { for } k=n, \\ \left(\frac{(k-1) \pi+\theta+\delta}{n}, \frac{(k+1) \pi+\theta-\delta}{n+1}\right) & \text { for } n+1 \leq k \leq 2 n .\end{cases}
$$

Observe that it is possible to take $\delta$ sufficiently small so that all these intervals are nonempty and $\delta<\theta$, and we assume that we have done so. (See Fig. 8.)

Claim. If $\varphi \in I_{k}(\delta)$ and $d \geq r$, then $z=d e^{i \varphi}$ does not lie on $C_{\theta}\left(g_{R}\right)$. That is, $C_{\theta}\left(g_{R}\right)$ does not cross the half-line $\left\{d e^{i \varphi}: d \geq r\right\}$.

To verify the claim, we must show that $\arg \left(g_{R}\left(d e^{i \varphi}\right)\right) \not \equiv \theta(\bmod \pi)$. Suppose first that $k \leq n$, so that $\varphi<\pi$. Then $\arg (z-R) \in(\varphi, \pi)$, and one computes that

$$
n \arg (z)+\arg (z-R) \in(k \pi+\theta+\delta,(k+1) \pi+\theta-\delta),
$$

and therefore

$$
\arg \left(g_{R}(z)\right) \in(k \pi+\theta,(k+1) \pi+\theta)
$$




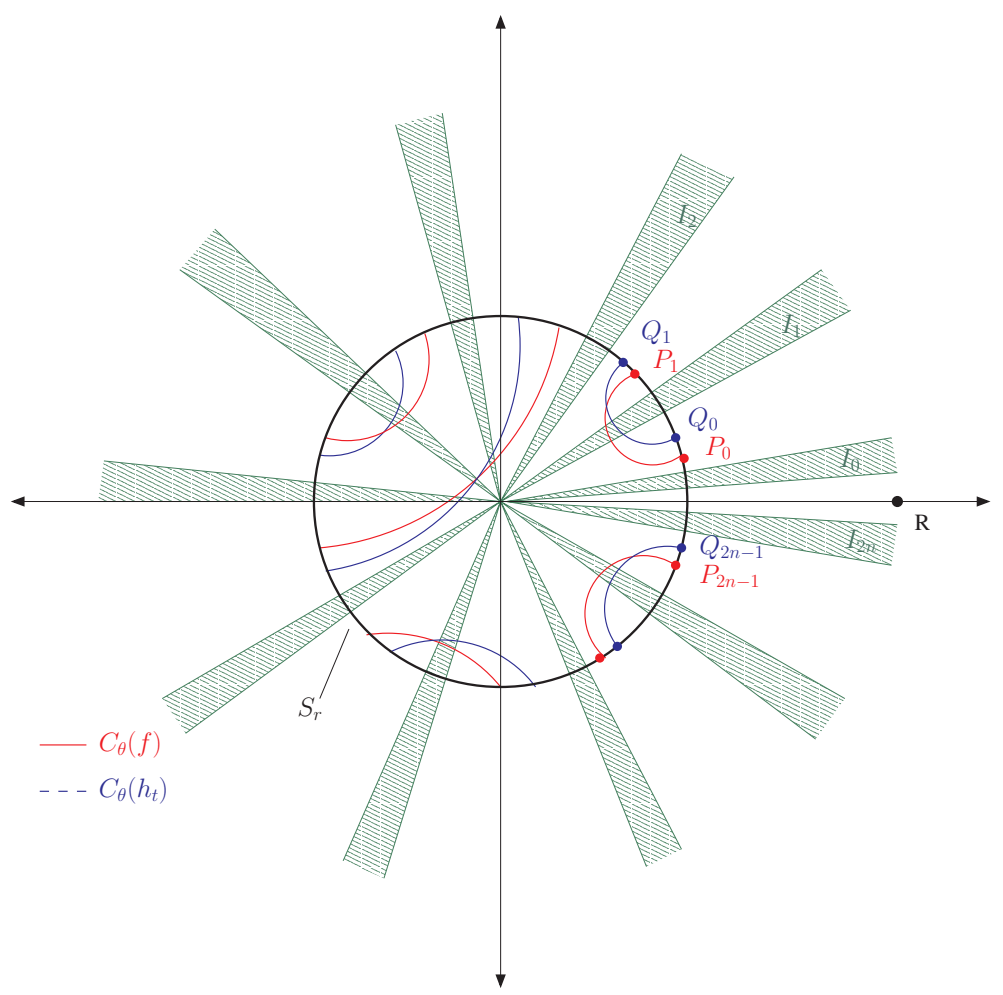

Fig. 8. Locating the new root $R$ between $I_{2 n}$ and $I_{0}$.

as desired. Similarly, if $k>n$, then $\varphi>\pi$ and $\arg (z-R) \in(\pi, \varphi)$, and we obtain the same conclusion about $\arg \left(g_{R}(z)\right)$.

Finally, we are in a position to complete the proof of Theorem 3.3. Choose $\varphi_{k} \in I_{k}(\delta)$ for each $0 \leq k \leq 2 n$. One checks that

$$
(k \pi+\theta) / n,((k+1) \pi+\theta) /(n+1) \in\left(\varphi_{k}+\delta /(n+1), \varphi_{k+1}-\delta /(n+1)\right)
$$

for $0 \leq k \leq 2 n-1$. Moreover, $\theta /(n+1)<\varphi_{0}$ and $((2 n+1) \pi+\theta) /(n+1)>\varphi_{2 n}$.

Since $\left|\arg \left(Q_{k}\right)-(k \pi+\theta) / n\right|<\delta /(n+1)$, we have established:

- $\arg \left(Q_{k}\right) \in\left(\varphi_{k}, \varphi_{k+1}\right)$,

- the unique $j$ such that $(j \pi+\theta) /(n+1) \in\left(\varphi_{k}, \varphi_{k+1}\right)$ is $j=k+1$, and

- $C_{\theta}\left(g_{R}\right)$ does not cross the half-lines $\left\{d e^{i \varphi_{k}}: d \geq r\right\}$ and $\left\{d e^{i \varphi_{k+1}}: d \geq r\right\}$.

Together, these facts imply that for $0 \leq k \leq 2 n-1$, the connected component of $C_{\theta}\left(g_{R}\right)$ containing $Q_{k}$ has an asymptote at angle $((k+1) \pi+\theta) /(n+1)$. This proves that if $a, b$ are matched in $M(f, \theta)=M\left(C_{\theta}\left(g_{R}\right), r\right)$, then $a+1, b+1$ are matched in $M\left(g_{R}, \theta\right)$. By elimination, 0 and $2 n+1$ must also be matched in $M\left(g_{R}, \theta\right)$; that is, $M\left(g_{R}, \theta\right)=\widehat{M(f, \theta)}$.

We can now complete the proof of the Inverse Basketball Theorem. 
Proof of Theorem 3.1. Suppose that $\alpha<\beta$. Let $f_{\eta}(z)$ be the monic polynomial $e^{i n \eta} f\left(e^{-i \eta} z\right)$. Then the curve $C_{n \eta+\theta}\left(f_{\eta}\right)$ is the rotation of $C_{\theta}(f)$ by $\eta$. Taking $\eta$ such that $n \eta+\beta<\pi$, we see that $B(f, \alpha, \beta)=B\left(f_{\eta}, 0, \beta-\alpha\right)$; we can therefore assume without loss of generality that $\alpha \neq 0$.

From the discussion following Proposition 2.10, recall that the set of all basketballs is closed under the rotation which replaces each quartet $\{i, j\},\{k, \ell\}$ with the quartet $\{i-2, j-2\},\{k-2, \ell-2\}$ (with subtraction taken modulo $4 n$ ). This transformation can also be realized on the set of basketballs $B(f, \alpha, \beta)$ arising from monic polynomials. Indeed, taking $\eta=-\pi / n$, we see that $a, b$ are matched in $M\left(f_{\pi / n}, \theta\right)$ if and only if $a-1, b-1$ are matched in $M(f, \theta)$. Since this is true for both $\theta=\alpha, \beta$, we see that $B\left(f_{\pi / n}, \alpha, \beta\right)$ is the rotation of $B(f, \alpha, \beta)$.

Recall also that the set of basketballs is closed under the half-rotation that replaces each quartet $\{i, j\},\{k, \ell\}$ with the quartet $\{k-1, \ell-1\},\{i-1, j-1\}$. Choose $0<$ $\gamma<\beta-\alpha$, and take $\eta=-(\alpha+\gamma) / n$. One checks similarly that the basketball $B\left(f_{\eta}, \beta-\alpha-\gamma, \pi-\gamma\right)$ is the half-rotation of $B(f, \alpha, \beta)$.

We proceed by induction on the degree of $f$, the case $n=1$ being trivial. Let $B$ be any basketball of order $n+1$. This contains an ear by Proposition 2.10, and by rotation we may assume that the ear contains the pair $\{4 n+3,1\}$. The other pair in this ear is either $\{4 n+2,0\}$ or $\{0,2\}$. If the ear is $\{4 n+3,1\},\{0,2\}$ then the half-rotation of $B$ contains the ear $\{4 n+3,1\},\{4 n+2,0\}$. By the observation in the previous paragraph, we may assume without loss of generality that the ear is $\{4 n+3,1\},\{4 n+2,0\}$.

Let $\left(M_{\alpha}, M_{\beta}\right)$ be the ordered pair of matchings on $[0,2 n+1]$ corresponding to $B$. By assumption, the pair $\{0,2 n+1\}$ is contained in both $M_{\alpha}$ and $M_{\beta}$.

For $\theta=\alpha, \beta$, let $M_{\theta}^{\prime}$ be the matching on [0,2n-1] such that $\{a, b\} \in M_{\theta}^{\prime}$ if and only if $\{a+1, b+1\} \in M_{\theta}$. Then the bimatching $B^{\prime}$ corresponding to $\left(M_{\alpha}^{\prime}, M_{\beta}^{\prime}\right)$ is a basketball of degree $n$, and so by the induction hypothesis $B^{\prime}=B(f, \alpha, \beta)$ for some monic polynomial $f(z)$. Since $\alpha \neq 0$, by Theorem 3.3 we see that $B$ is the basketball of $(z-R) f(z)$ for $R$ sufficiently large, and we are done.

\section{Necklaces of Matchings}

More generally, we are interested in classifying the possibilities for the topology of the family

$$
\mathcal{C}(f)=\left\{C_{\theta}(f): \theta \in \mathbb{R} / \pi \mathbb{Z}\right\},
$$

which is truly an invariant of $f$ itself, not depending on a choice of angle. The family $\mathcal{C}(f)$ is fibered over the base $\mathbb{R} / \pi \mathbb{Z}$ with fiber $C_{\theta}(f)$ above $\theta$. In particular, suppose that $f(z)$ has distinct roots. Let $z_{1}, \ldots, z_{n-1}$ be the roots of $f^{\prime}$, and suppose further that $\arg f\left(z_{1}\right), \ldots, \arg f\left(z_{n-1}\right)$ are distinct. Then the family $\mathcal{C}(f)$ has $n-1$ fibers with ordinary singularities, and is smooth elsewhere; that is, it is smooth over $n-1$ open $\operatorname{arcs} \mathcal{A}_{i}$ arranged cyclically around the circle $\mathbb{R} / \pi \mathbb{Z}$. The noncrossing matching $M(f, \theta)$ is the same for all $\theta \in \mathcal{A}_{i}$; denote it by $M_{i}$. We thus obtain an $(n-1)$-tuple $\mathcal{M}=\left(M_{1}, \ldots, M_{n-1}\right)$ of noncrossing matchings, and the data of $\mathcal{M}$ determines the topology of $\mathcal{C}(f)$.

Let $M_{n}$ be the matching $\left\{\{i-1, j-1\}:\{i, j\} \in M_{1}\right\}$, taking all indices modulo $2 n$ as usual. Then the $(n-1)$-tuple of matchings $\left(M_{1}, \ldots, M_{n-1}\right)$ has the following property: 
for $0 \leq t \leq n-1$, the matching $M_{t+1}$ can be obtained from $M_{t}$ by taking a suitable pair of pairs $\{i, j\},\{k, \ell\}$ and replacing them with $\{i, \ell\},\{j, k\}$. Call an $(n-1)$-tuple of noncrossing matchings possessing this property a necklace of matchings of order $n$. For example, from this point of view the basketball of a quadratic polynomial $f$ is determined by the two possibilities for the necklace $\left(M_{1}\right)$, and by whether $\arg f\left(z_{1}\right) \quad(\bmod \pi)$ is greater or smaller than $\pi / 2$ (see also Remark 1.7 ).

It is natural to ask whether every necklace of order $n$ must arise from a polynomial of degree $n$. To that end, define a multi-ear to be an integer $i$ for which $\{i, i+1\} \in$ $M_{1}, \ldots, M_{t}$ and $\{i-1, i\} \in M_{t+1}, \ldots, M_{n}$ for some $t$. If every necklace contains a multi-ear, then Theorem 3.3 can be used to show that every necklace arises from a polynomial; otherwise, new techniques will be necessary. We note the following result, obtained via exhaustive computer calculation:

Proposition 4.1. For $n \leq 8$, we have:

(1) Every necklace of order $n$ contains a multi-ear.

(2) If $\mathcal{M}=\left(M_{1}, \ldots, M_{n-1}\right)$ is a necklace of order $n$ and $M_{n}$ is defined as above, then for all $1 \leq t<u \leq n$ the bimatching corresponding to $\left(M_{t}, M_{u}\right)$ is a basketball.

(3) The number of necklaces of order $n$ is $2(2 n)^{n-2}$.

We remark that (1) implies (2). Indeed, (1) implies that every necklace of order at most 8 arises from a polynomial, and (2) is automatically satisfied by any necklace arising from a polynomial. The numbers $2(2 n)^{n-2}$ appear as sequence A097629 in [11], enumerating unrooted directed trees on $n$ vertices; we do not know a bijective reason that this sequence should also count necklaces of matchings.

\section{Acknowledgments}

It is our pleasure to thank Mira Bernstein, Pete Clark, Valery Liskovets, Grisha Mikhalkin, Vic Reiner, and Timothy Walsh for helpful conversations. We began our work on this project while the first two authors were instructors and the third author was a student at Canada/USA Mathcamp, a summer program for high school students. During much of the time that this work was ongoing, the first two authors were postdoctoral fellows at the University of Minnesota and McGill University, respectively.

\section{References}

1. Lars Ahlfors, Complex Analysis, 3rd edn., McGraw-Hill, New York, 1979.

2. Jean Le Rond d'Alembert, Recherches sur le calcul integral, Hist. Acad. Sci. Berlin 2 (1746), 182-224.

3. Jacek Bochnak, Michel Coste, and Marie-Françoise Roy, Real Algebraic Geometry, Springer-Verlag, Berlin, 1998.

4. Paul H. Edelman, Chain enumeration and non-crossing partitions, Discrete Math. 31 (1980), 171-180.

5. Carl Friedrich Gauss, Demonstratio nova theorematis omnem functionem algebraicam rationalem integram unius variabilis in factores reales primi vel secundi gradus resolvi posse, Ph.D. thesis, Universität Helmstedt, 1799. 
6. Steve M. Gersten and John R. Stallings, On Gauss's first proof of the fundamental theorem of algebra, Proc. Amer. Math. Soc. 103(1) (1988), 331-332.

7. R.W. Goldbach and Robert Tijdeman, Pairings of $2 n$ points on a circle, Util. Math. 38 (1990), 277-284.

8. Ilia Itenberg and Oleg Viro, Patchworking algebraic curves disproves the Ragsdale conjecture, Math. Intelligencer 18(4) (1996), 19-28.

9. Valery A. Liskovets and Timothy R. Walsh, Counting unrooted maps on the plane, Adv. Appl. Math., 36(4) (2006), 364-387.

10. Rodica Simion, Noncrossing partitions, Discrete Math. 317 (2000), 367-409.

11. Neil J.A. Sloane, The On-Line Encyclopedia of Integer Sequences, published electronically at http://www.research.att.com/ njas/sequences/.

12. Richard P. Stanley, Enumerative Combinatorics, vol. 2, Cambridge University Press, Cambridge, 1999.

13. John Stillwell, Mathematics and Its History, Springer-Verlag, New York, 1989.

14. Oleg Ya. Viro, Gluing of plane real algebraic curves and constructions of curves of degrees 6 and 7 , Topology (Leningrad, 1982), Lecture Notes in Mathematics, 1060, Springer, Berlin, 1984.

15. Oleg Ya. Viro, Achievements in the topology of real algebraic varieties in the last six years, Russian Math. Surveys 41(3) (1986), 55-82.

Received November 9, 2005, and in revised form February 14, 2006. Online publication February 9, 2007. 